\title{
Communication
}

\section{On the Theoretical Maximum Directivity of a Radiating Aperture From Modal Field Expansions}

\author{
Lucas Polo-López ，Juan Córcoles ，Jorge A. Ruiz-Cruz ，José R. Montejo-Garai ， and Jesús M. Rebollar
}

\begin{abstract}
This communication addresses the maximization of the directivity from a radiating aperture through the derivation of a closedform expression for the modal field amplitudes whose field distribution at the aperture maximizes the directivity in a desired direction. This closedform expression arises from a convex formulation of the maximization problem (as a ratio of two Hermitian forms), which mathematically ensures that the achieved directivity value is the global maximum for a specified number of propagating modes. The proposed technique, which is valid for any aperture shape and polarization, is discussed and compared with previous work. Several numerical examples considering different main lobe directions, aperture geometries, and polarizations are presented. To verify the numerical results, radiation patterns and directivity values are computed using both commercial tools and in-house developed software.
\end{abstract}

Index Terms-Antenna radiation pattern synthesis, apertures, directivity, modal field, optimization methods.

\section{INTRODUCTION}

Aperture antennas are one of the most practical antennas for microwave and millimeter-wave systems [1]. These antennas can take several forms, such as open-ended waveguides, slots, or horns among others. Nevertheless, an appropriate modeling and design of any of them lies on a good understanding of the radiating aperture problem [2]. Since one of the most important parameters of an antenna is its directivity value, maximizing the directivity achieved by a certain topology or dimensions becomes one of the main challenges in antenna engineering [3], [4].

In this communication, a closed-form expression for directivity maximization in aperture antennas is derived and discussed. After the formulation is presented, some application cases, where the method is compared with previous work [2], [5], are shown. The achieved numerical results are simulated with both commercial and in-house developed software.

The proposed formulation presents two main advantages. First, it can be applied to any aperture geometry, for any direction of maximization and polarization. Second, it can be formally guaranteed that, for a certain number of modes and aperture size, the achieved directivity value is the highest possible.

The key idea of the proposed formulation is expressing the directivity of a radiating aperture as a ratio of two Hermitian forms [6], which

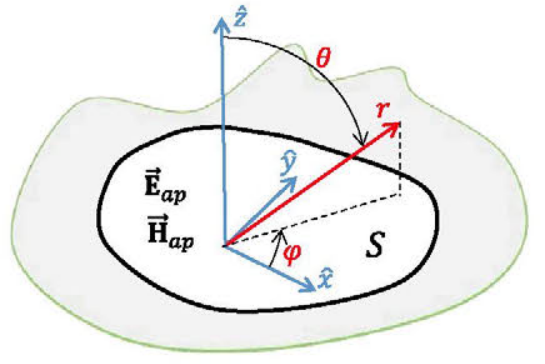

Fig. 1. Representation of the coordinate system used to compute the radiation patterns of an aperture placed at the $x y$-plane. The gray area represents the boundary conditions imposed by the used equivalence principle out of the aperture at the $x y$-plane.

can be easily maximized. In the field of antenna design, this idea has already been exploited for the optimization of array excitation coefficients [7], [8], and the present contribution shows how it can be applied to radiating apertures. Therefore, this communication aims to set the theoretical framework of the optimum modal amplitudes for a general-shaped radiating aperture, taking the particularities of modal expansions into account.

\section{Formulation}

In an aperture antenna, as the one shown in Fig. 1 lying on the $x y$-plane, the radiated field can be computed from the transverse electric (TE) and transverse magnetic (TM) fields at the aperture $\overrightarrow{\mathbf{E}}_{a p}(x, y), \overrightarrow{\mathbf{H}}_{a p}(x, y)$. These fields can be written as a summation of modes corresponding to a waveguide with a transverse section equal to the aperture shape [9]

$$
\left\{\begin{array}{l}
\overrightarrow{\mathbf{E}}_{a p}(x, y) \\
\overrightarrow{\mathbf{H}}_{a p}(x, y)
\end{array}\right\}=\sum_{m=1}^{M} c_{m}\left\{\begin{array}{l}
\overrightarrow{\mathbf{e}}_{m}(x, y) \\
\overrightarrow{\mathbf{h}}_{m}(x, y)
\end{array}\right\}
$$

where $c_{m}$ represents the complex amplitudes of the electric $\left(\overrightarrow{\mathbf{e}}_{m}\right)$ and magnetic $\left(\overrightarrow{\mathbf{h}}_{m}\right)$ fields of the $m$ th mode, which can be computed as

$$
\begin{gathered}
\overrightarrow{\mathbf{e}}_{m}(x, y)=Z_{m}^{\frac{1}{2}} \begin{cases}\nabla_{t} \Phi_{m}(x, y) \times \hat{\mathbf{z}} & \text { TE Modes } \\
\nabla_{t} \Phi_{m}(x, y) & \text { TM Modes }\end{cases} \\
\overrightarrow{\mathbf{h}}_{m}(x, y)=Y_{m}^{\frac{1}{2}} \begin{cases}\nabla_{t} \Phi_{m}(x, y) & \text { TE Modes } \\
\hat{\mathbf{z}} \times \nabla_{t} \Phi_{m}(x, y) & \text { TM Modes. }\end{cases}
\end{gathered}
$$

These transversal fields can be expressed with a scalar, real, and frequency-independent function $\Phi_{m}(x, y)$, which is proportional to the longitudinal component of the electric or magnetic field, depending on whether the $m$ th mode is TM or TE. The term $Z_{m}=Y_{m}^{-1}$ is the mode impedance [9] of the $m$ th mode. Please note that the expansions in (1) should have, in general, an infinite number of terms. However, in this communication, only the $M$ propagating modes will be considered to contribute to the radiated field [5]. 
The far field radiated by an aperture $\overrightarrow{\boldsymbol{E}}_{\text {rad }}(\theta, \phi)$ can be computed using one of the well-known equivalence principles [10], denoted by the operator $\mathcal{P}$, as

$$
\overrightarrow{\boldsymbol{E}}_{\text {rad }}(\theta, \phi)=\mathcal{P}\left[\overrightarrow{\mathbf{E}}_{a p}(x, y), \overrightarrow{\mathbf{H}}_{a p}(x, y)\right] .
$$

In the case of using both electric and magnetic currents (other options with only electric or only magnetic currents are also possible [10]), the equivalence principle $\mathcal{P}$ in terms of $(\theta, \phi)$ and $(x, y)$ components takes the form

$$
\begin{gathered}
E_{\theta}(\theta, \phi)=j \frac{k_{0}}{4 \pi}\left(P_{x} \cos \phi+P_{y} \sin \phi\right. \\
\left.-\eta_{0} \cos \theta\left(Q_{x} \sin \phi-Q_{y} \cos \phi\right)\right) \\
E_{\phi}(\theta, \phi)=-j \frac{k_{0}}{4 \pi}\left(\cos \theta\left(P_{x} \sin \phi-P_{y} \cos \phi\right)\right. \\
\left.+\eta_{0}\left(Q_{x} \cos \phi+Q_{y} \sin \phi\right)\right)
\end{gathered}
$$

where $P_{x}, P_{y}$ and $Q_{x}, Q_{y}$ are the $(\theta, \phi)$-dependent functions computed from the surface integral of the $x$ and $y$ components of the electric and magnetic fields at the aperture surface $S$ (see Fig. 1) [10]

$$
\left\{\begin{array}{c}
P x \\
y \\
Q_{x}
\end{array}\right\}=\iint_{S}\left\{\begin{array}{l}
\mathrm{E}_{a p}, \underset{y}{x}\left(x^{\prime}, y^{\prime}\right) \\
\mathrm{H}_{a p,}, \underset{y}{x}\left(x^{\prime}, y^{\prime}\right)
\end{array}\right\} \mathrm{e}^{j k_{0}\left(u x^{\prime}+v y^{\prime}\right)} d x^{\prime} d y^{\prime}
$$

$k_{0}$ being the free-space wavenumber, $u=\sin \theta \cos \phi$, and $v=$ $\sin \theta \sin \phi$. Please note that to compute the actual electric field at every point of the space, the expressions in (5) must be multiplied by $\mathrm{e}^{-j k_{0} r} / r$.

Now, by inserting the modal expansion (1) into (6) and substituting in the equivalence principle (4), the field radiated by each mode $\vec{f}_{m}(\theta, \phi)$ can be computed independently, and thus, the total radiated field is obtained from the combination of $\vec{f}_{m}(\theta, \phi)$ weighted by modal complex amplitudes $c_{m}$ as

$$
\overrightarrow{\boldsymbol{E}}_{\text {rad }}(\theta, \phi)=\sum_{m=1}^{M} c_{m} \underbrace{\mathcal{P}\left[\overrightarrow{\mathbf{e}}_{m}(x, y), \overrightarrow{\mathbf{h}}_{m}(x, y)\right]}_{\vec{f}_{m}(\theta, \phi)} .
$$

It should be noted that in the computation of $\vec{f}_{m}(\theta, \phi)$, the equivalence principle is applied to the $m$ th modal electric $\overrightarrow{\mathbf{e}}_{m}$ and magnetic $\overrightarrow{\mathbf{h}}_{m}$ fields at the aperture. In this specific case, since $\overrightarrow{\mathbf{e}}_{m}$ and $\overrightarrow{\mathbf{h}}_{m}$ are related through the mode impedance $\left(Z_{m}\right)$, it is known that the following relations hold for the surface integrals of the $m$ th mode in (5) and (6):

$$
Q_{x, m}=-P_{y, m} / Z_{m}, \quad Q_{y, m}=P_{x, m} / Z_{m} .
$$

Thus, only two integrals must be calculated, which can be used to alleviate the computational burden.

The copolar directivity $D_{c p}$ of an antenna for a given polarization [11] is defined as the ratio of the radiation intensity corresponding to the copolar component of the far field $E_{c p}(\theta, \phi)$ and the total radiation intensity averaged over all directions [12], which is more commonly written as

$$
D_{c p}(\theta, \phi)=\frac{\left|E_{c p}(\theta, \phi)\right|^{2} / 2 \eta_{0}}{P_{r a d} / 4 \pi}
$$

where $\eta_{0}$ is the impedance of free space and $P_{r a d}$ is the power radiated by the antenna. From (7), the overall copolar radiated field can be written as a summation of the copolar fields radiated by each mode as

$$
E_{c p}(\theta, \phi)=\sum_{m=1}^{M} c_{m} f_{m, c p}(\theta, \phi) .
$$

Then, by defining the column vector $\mathbf{f}$ as

$$
\mathbf{f}(\theta, \phi)=\left[\begin{array}{c}
f_{1, c p}^{*}(\theta, \phi) \\
f_{2, c p}^{*}(\theta, \phi) \\
\vdots \\
f_{M, c p}^{*}(\theta, \phi)
\end{array}\right]
$$

where superscript $*$ stands for the complex conjugate, the absolute value of the copolar radiation pattern can be rewritten using matrix notation as

$$
\left|E_{c p}(\theta, \phi)\right|^{2}=\mathbf{c}^{H} \mathbf{f}(\theta, \phi) \mathbf{f}(\theta, \phi){ }^{H} \mathbf{c}=\mathbf{c}^{H} \mathbf{F}(\theta, \phi) \mathbf{c}
$$

where $\mathbf{c}$ is the column vector with the $M$ modal amplitudes $c_{m}$ and superscript $H$ denotes the Hermitian operator (transpose conjugate).

The radiated power can be computed in terms of the electromagnetic field at the aperture [13] from

$$
P_{\text {rad }}=\frac{1}{2} \iint_{S} \hat{\mathbf{z}} \cdot \Re\left\{\overrightarrow{\mathbf{E}}_{a p} \times \overrightarrow{\mathbf{H}}_{a p}^{*}\right\} \mathrm{d} S .
$$

Now, by inserting (1)-(3) into (13), the radiated power can be expressed in matrix notation as

$$
P_{\text {rad }}=\frac{1}{2} \mathbf{c}^{H} \mathbf{A c}, A_{m n}= \begin{cases}\iint_{S}\left|\nabla_{t} \Phi_{m}\right|^{2} \mathrm{~d} S & m=n \\ 0 & m \neq n\end{cases}
$$

where the diagonal nature of matrix $\mathbf{A}$ arises from the well-known modal fields orthogonality [9].

Finally, by inserting (12) and (14) into (9), the copolar directivity in a certain direction $\left(\theta_{0}, \phi_{0}\right)$ can be expressed as

$$
D_{c p}\left(\theta_{0}, \phi_{0}\right)=\frac{\left(\mathbf{c}^{H} \mathbf{f}_{0} \mathbf{f}_{0}^{H} \mathbf{c}\right) / 2 \eta_{0}}{\left(\mathbf{c}^{H} \mathbf{A c}\right) / 8 \pi}=\frac{4 \pi}{\eta_{0}} \frac{\mathbf{c}^{H} \mathbf{F}_{0} \mathbf{c}}{\mathbf{c}^{H} \mathbf{A c}}
$$

where $\mathbf{f}_{0}$ and $\mathbf{F}_{0}$ are the result of particularizing, respectively, $\mathbf{f}(\theta, \phi)$ and $\mathbf{F}(\theta, \phi)$ for the $\left(\theta_{0}, \phi_{0}\right)$ direction.

It can be noted that (15) is a ratio of two Hermitian forms which, according to a long-known theorem in matrix algebra [6], is maximum for the eigenvector $\mathbf{c}_{\max }$ corresponding to the largest eigenvalue $\lambda_{\max }$ of the generalized eigenvalue problem $\left(\mathbf{F}_{0}-\lambda \mathbf{A}\right) \mathbf{c}=\mathbf{0}$. Indeed, since $\mathbf{F}_{0}$ is a rank-1 matrix, $\lambda_{\max }$ is the only nonzero eigenvalue, and the corresponding eigenvector takes the form $\mathbf{c}_{\max }=\kappa \mathbf{A}^{-1} \mathbf{f}_{0}$ [7], where $\kappa$ is an arbitrary complex constant that does not affect the relative amplitude distribution. Therefore, the complex amplitude for the $m$ th mode, which maximizes the copolar directivity in a specified $\left(\theta_{0}, \phi_{0}\right)$ direction, can be written in a compact closed-form expression as

$$
c_{m}=\kappa \frac{f_{m, c p}^{*}\left(\theta_{0}, \phi_{0}\right)}{\iint_{S}\left|\nabla_{t} \Phi_{m}\right|^{2} \mathrm{~d} S}, \quad m=1, \ldots, M .
$$

One of the main advantages of the presented formulation is that it does not make any assumptions related to the direction for which the directivity is maximized $\left(\theta_{0}, \phi_{0}\right)$, the aperture shape or the excited modal family at the aperture (i.e., all the propagating modes supported by the aperture geometry could be used for the synthesis or only a subset of them if this is desired). Additionally, it should be emphasized that thanks to the aforementioned theorem, it can be ensured that the obtained directivity value is the global maximum for the defined aperture geometry and the specified number of modes.

\section{BORESIGHT DIRECTIVITY MAXIMIZATION}

\section{A. Comparison With Previous Work}

This particular case [maximizing the directivity for the $\left(\theta_{0}, \phi_{0}\right)=$ $(0,0)$ direction, see Fig. 1] is a usual problem in antenna engineering 
with some recent advances described in [5]. The technique proposed in [5] is based on obtaining a field distribution in the aperture as close as possible to a unidirectional and uniform field, $E_{0} \hat{\mathbf{x}}$, by projecting it onto the different modes in the aperture. This approach will allow to test our formulation for the classic scenario of achieving maximum directivity for $\left(\theta_{0}, \phi_{0}\right)=(0,0)$ with linear polarization, as in [5]. Other novel test cases not considered in this previous work will be addressed in Section III-B.

The equivalence principle used in [5] only considers magnetic currents

$$
\begin{aligned}
& E_{\theta}(\theta, \phi)=j \frac{k_{0}}{2 \pi}\left(P_{x} \cos \phi+P_{y} \sin \phi\right) \\
& E_{\phi}(\theta, \phi)=-j \frac{k_{0}}{2 \pi}\left(\cos \theta\left(P_{x} \sin \phi-P_{y} \cos \phi\right)\right) .
\end{aligned}
$$

The radiated field in the boresight direction is computed by inserting (6) into (17) for $(\theta, \phi)=(0,0)$. Provided that the copolar direction is defined as $\hat{\mathbf{x}}$ (the same as $\hat{\boldsymbol{\theta}}$ in $\theta=0, \phi=0$ ), the values of $c_{m}$ can be obtained from $P_{x, m}^{*}(0,0)$ as

$$
c_{m}=\kappa \frac{-j k_{0}}{2 \pi} \frac{\iint_{S} \hat{\mathbf{x}} \cdot \overrightarrow{\mathbf{e}}_{m}^{*} \mathrm{~d} S}{\iint_{S}\left|\nabla_{t} \Phi_{m}\right|^{2} \mathrm{~d} S}=\kappa \frac{-j k_{0}}{2 \pi} Z_{m}^{\frac{1}{2}} \frac{\iint_{S} \frac{\partial \Phi_{m}}{\partial y} \mathrm{~d} S}{\iint_{S}\left|\nabla_{t} \Phi_{m}\right|^{2} \mathrm{~d} S}
$$

which leads to the same formal expression given in [5] following a projection approach. Please note that the particularizations that have been made only regard the direction of maximum radiation $(\theta, \phi)=(0,0)$ and the polarization (linear in $x)$ of the radiated field. Besides that, (18) is valid for any aperture shape. It should also be noted that, as demonstrated in [5], the value of $c_{m}$ in (18) is zero when evaluated for a TM modal function.

As a specific example, a circular aperture of radius $a$ is considered, with a set of modal field functions denoted by $T E_{q r}^{c / s}, T M_{q r}^{c / s}$, where $q$ and $r$ are, respectively, the number of angular and radial variations and $c / s$ accounts for the two different trigonometric functions $\cos / \sin$ of $\Phi_{m}$ in the circular waveguide [9]. For the case studied in [5] with $q=1$, (18) can be computed analytically, resulting in

$$
c_{m}= \begin{cases}\frac{-j k_{0}}{2 \pi} Z_{m}^{\frac{1}{2}} a \sqrt{\frac{2 \pi}{p_{1 m}^{\prime 2}-1}} & \text { TE Modes } \\ 0 & \text { TM Modes }\end{cases}
$$

where $p_{1 m}^{\prime}$ are the zeros of the derivative of the first-order Bessel function. It can be clearly seen that this result leads to the same relative amplitude distribution of [5, eq. (15)]. The term $-j k_{0} /(2 \pi)$ in (19) does not affect the relative distribution of the amplitudes. The term $Z_{m}^{\frac{1}{2}}$ is explained by the fact that the amplitudes in the modal series used in this communication have a relation of $Z_{m}^{\frac{1}{2}}$ with the amplitudes used in [5].

\section{B. Numerical Examples}

The developed formulation is illustrated with four numerical examples. The first one consists of a circular aperture radiating a linearly polarized field. The chosen frequency is $f_{0}=12 \mathrm{GHz}$, and the radius of the aperture is $a=2 \lambda_{0}$, which is a size used in the typical horn antenna designs. All $T E_{q r}^{c, s}, T M_{q r}^{c, s}$ propagating modes in the equivalent circular waveguide (which are 77) are considered in the directivity maximization process. Since the studied apertures are electrically large, this choice of the number of modes is enough to represent the aperture field for the directivity maximization while discarding the evanescent modes and avoiding the fast-oscillating spatial nature of high cutoff frequency modes and small amplitudes. The obtained modal amplitudes (normalized to that of the first mode,
TABLE I

COMPlex MOdAl Amplitudes of THE Circul ar APERTUREEXAMPLES

\begin{tabular}{|lr|lr|}
\hline Mode & Linear polarization & Mode & Circular polarization \\
\hline$T E_{11}^{c}$ & 1 & $T E_{11}^{c}$ & 1 \\
$T E_{12}^{c}$ & -0.2956 & $T E_{11}^{s}$ & $j$ \\
$T E_{13}^{c}$ & 0.1845 & $T E_{12}^{c}$ & -0.2956 \\
$T E_{14}^{c}$ & -0.1497 & $T E_{12}^{s}$ & $-j 0.2956$ \\
& & $T E_{13}^{c}$ & 0.1845 \\
& & $T E_{13}^{s}$ & $j 0.1845$ \\
& & $T E_{14}^{c}$ & -0.1497 \\
& & $T E_{14}^{s}$ & $-j 0.1497$ \\
\hline
\end{tabular}

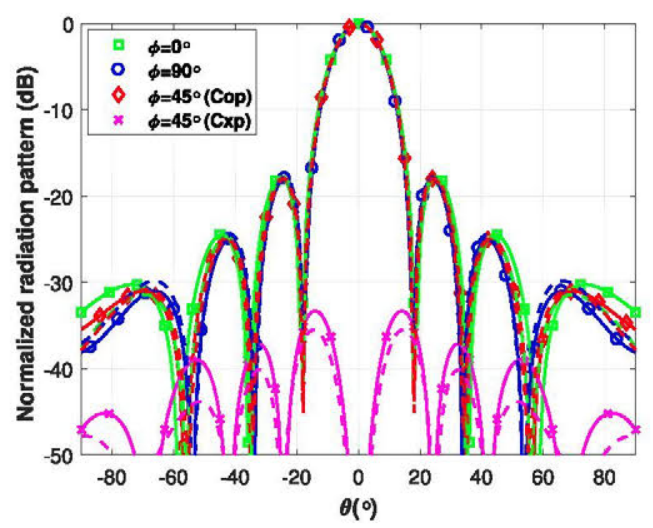

Fig. 2. Normalized radiation pattern of a linearly polarized circular radiating aperture. Solid line: this communication, with $D_{0}=21.80 \mathrm{dBi}$. Dashed line: CST, with $D_{0}=22.00 \mathrm{dBi}$.

TABLE II

DiRECTIVITy (dBi) ACHIEVED By APERTURES OF DIFFERENT RADII

\begin{tabular}{|c|c|c|c|c|c|}
\hline Radius $a$ & $1 \lambda_{0}$ & $2 \lambda_{0}$ & $3 \lambda_{0}$ & $4 \lambda_{0}$ & $5 \lambda_{0}$ \\
\hline Proposed formulation & 15.60 & 21.80 & 25.38 & 27.91 & 29.87 \\
\hline Ideal case & 15.96 & 21.98 & 25.51 & 28.00 & 29.94 \\
\hline
\end{tabular}

i.e., $c_{m} / c_{1}$ ) can be found in the first column of Table I. Only the modes with nonzero amplitude are shown in Table I, leading to the expected result that only TE modes of the considered polarization with $q=1$ have nonzero values.

The radiation pattern generated by these amplitudes is shown in Fig. 2. It has been computed using (5) and (7) along with the modal amplitudes of Table I. Additionally, the radiation pattern has also been computed using a commercial tool (CST Microwave Studio). The results obtained by the commercial tool are superimposed in Fig. 2 over the ones from this communication. It can be seen how they are in good agreement not only in the normalized radiation pattern but also in the achieved directivity value.

An aperture with a greater radius $a$ has the potential to achieve a higher directivity value. However, the actual directivity will depend on the field distribution. Therefore, a larger aperture may provide a lower directivity than a different physically smaller aperture with a more appropriate mode combination. To study the effect of the aperture radius, several apertures have been optimized. In each case, all the propagating modes in the equivalent waveguide have been used. It should be noted that, for the same operating frequency, the number of propagating modes increases with the aperture size. Table II shows the achieved directivity compared with the value of the ideal case, which corresponds to an aperture with a unidirectional 


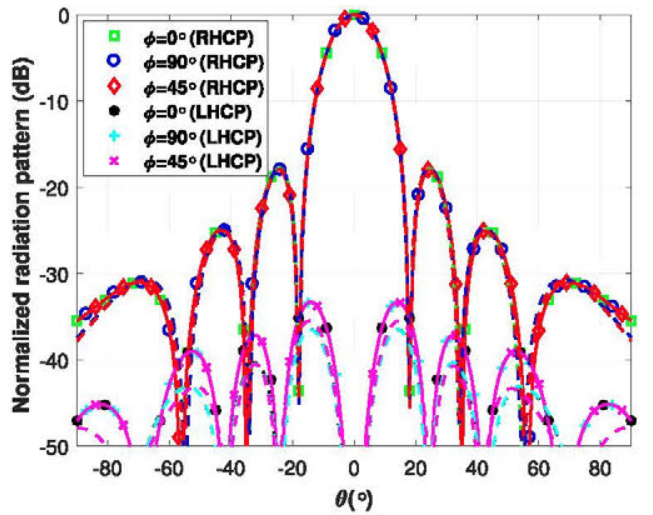

Fig. 3. Normalized radiation pattern of a circularly polarized circular radiating aperture. Solid line: this communication, with $D_{0}=21.80 \mathrm{dBi}$. Dashed line: CST, with $D_{0}=22.00 \mathrm{dBi}$.

and uniform electric field. In this situation, the aperture efficiency is maximum. The directivity for this case can be computed analytically using the well-known [1], [10] expression $D_{\text {ideal }}=\left(2 \pi a / \lambda_{0}\right)^{2}$. The results presented in Table II show that an aperture efficiency close to 1 is achieved since the obtained directivity values are very similar to those of the ideal case. This is in accordance with the presented formulation since it guarantees the global optimum, thus providing the largest possible directivity value for a given set of propagating modes.

In the next example, the same circular aperture $\left(a=2 \lambda_{0}\right)$, with the same modal set, will be used to generate right-hand circular polarization (RHCP). The achieved modal amplitudes can be found in the second column of Table I (only the nonzero values are shown). All $T E_{q r}^{c / s}, T M_{q r}^{c / s}$ propagating modes, as in the linear polarization case, were used in the problem for checking purposes computing the amplitudes directly from (16). The formulation leads to the expected result, requiring modes with the same amplitude and $90^{\circ}$ phase difference. The obtained radiation pattern can be found in Fig. 3. It can be noted that it is difficult to distinguish the traces corresponding to the different cuts of the radiation pattern. This is due to the fact that the radiation pattern exhibits rotational symmetry, and therefore, the traces of the different cuts exactly overlap.

The formulation can also be applied to arbitrary-shaped apertures (see Section V), but, first, it has been checked with other classical shapes such as the square aperture of the following example. The directivity in two different cases (one with linear polarization along $\hat{\mathbf{y}}$ and the other with RHCP) will be maximized. The square aperture of the example has a side of $4 \lambda_{0}$ at $f_{0}=12 \mathrm{GHz}$. In this situation, there are 98 propagating modes, and a priori, all of them are considered for the maximization process. Nevertheless, when using (16) to compute the modal amplitudes, it is found that (as it happened with the circular aperture example) most of them are zero-valued. The obtained nonzero modal amplitudes (normalized to that of the first mode) can be found in Table III. Using these amplitudes, the obtained radiation patterns have been computed in the same way as in the circular aperture example. They are represented in Fig. 4 (linearly polarized case) and Fig. 5 (circularly polarized case).

\section{BEAM SCANNING}

One of the advantages of the proposed formulation is that the directivity can be maximized in any desired $(\theta, \phi)$ direction. This can be used to generate an off-boresight radiation pattern. A radiation pattern pointing to $(\theta, \phi)=\left(30^{\circ}, 0^{\circ}\right)$ with linear polarization along $\hat{\mathbf{y}}$ for a circular aperture is now studied. The same radiating aperture
TABLE III

Complex Modal Amplitudes of THE SQUARED APERTURE EXAMPles

\begin{tabular}{|rr|rr|}
\hline Mode & Linear polarization & Mode & Circular polarization \\
\hline$T E_{10}$ & 1 & $T E_{10}$ & 1 \\
$T E_{30}$ & 0.3336 & $T E_{01}$ & $-j$ \\
$T E_{50}$ & 0.2015 & $T E_{30}$ & 0.3336 \\
$T E_{70}$ & 0.1523 & $T E_{03}$ & $-j 0.3336$ \\
& & $T E_{50}$ & 0.2015 \\
& & $T E_{05}$ & $-j 0.2015$ \\
& & $T E_{70}$ & 0.1523 \\
& & $T E_{07}$ & $-j 0.1523$ \\
\hline
\end{tabular}

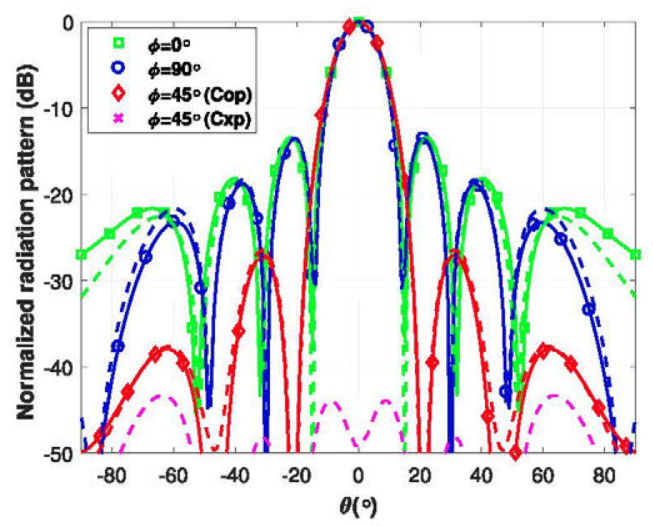

Fig. 4. Normalized radiation pattern of a linearly polarized square radiating aperture. Solid line: this communication, with $D_{0}=22.83 \mathrm{dBi}$. Dashed line: CST, with $D_{0}=22.98 \mathrm{dBi}$.

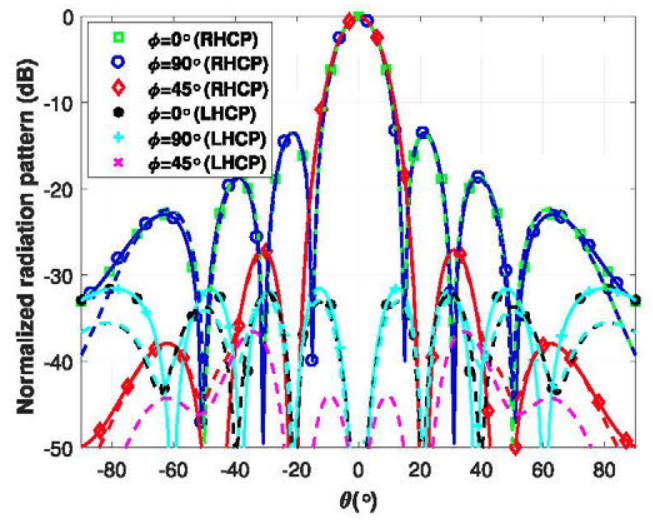

Fig. 5. Normalized radiation pattern of a circularly polarized square radiating aperture. Solid line: this communication, with $D_{0}=22.83 \mathrm{dBi}$. Dashed line: CST, with $D_{0}=22.98 \mathrm{dBi}$.

( $f_{0}=12 \mathrm{GHz}, a=2 \lambda_{0}$, and 77 propagating modes $)$ from Section III is considered. The normalized radiation pattern after the maximization process is shown in Fig. 6.

The next example shows that scanning is also possible for a radiation pattern with circular polarization. The aperture geometry and the operating frequency are the same as in the previous example, and in this case, the main lobe will be scanned to the $(\theta, \phi)=$ $\left(40^{\circ}, 0^{\circ}\right)$ direction with RHCP. The obtained radiation pattern is shown in Fig. 7.

\section{NONCANONICAL APERTURES}

Another main advantage provided by the developed formulation is that it can be applied to apertures with an arbitrary shape, as long 


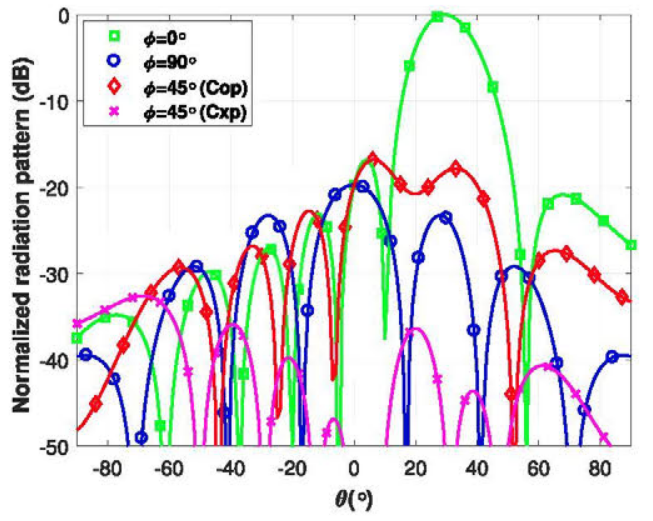

Fig. 6. Normalized radiation pattern of the beam-scanning example with linear polarization. The main lobe has been scanned to $\left(\theta_{0}, \phi_{0}\right)=\left(30^{\circ}, 0^{\circ}\right)$. $D_{0}=21.11 \mathrm{dBi}$.

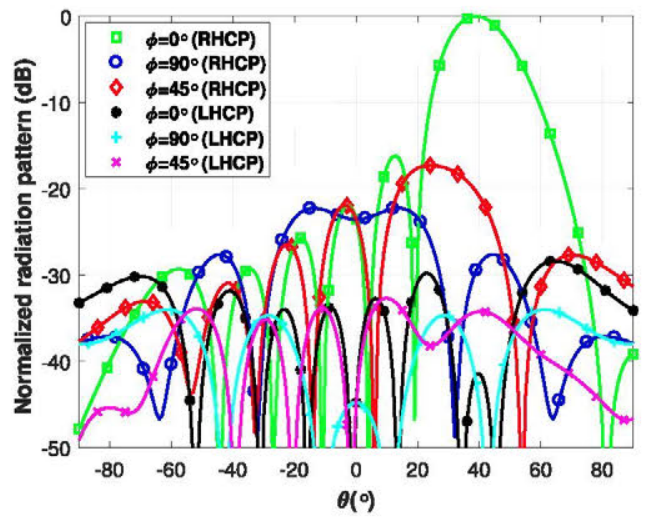

Fig. 7. Normalized radiation pattern of the beam-scanning example with circular polarization. The main lobe has been scanned to $\left(\theta_{0}, \phi_{0}\right)=\left(40^{\circ}, 0^{\circ}\right)$. $D_{0}=20.51 \mathrm{dBi}$

as their TE/TM modal field functions are available. In some cases, like the examples shown in Sections III and IV, the aperture field can be integrated analytically to compute (6) and (14). Nevertheless, other noncanonical apertures with modal field functions evaluated numerically can also be used in the proposed formulation to maximize the directivity from this type of apertures. Nowadays, the modal fields $\left(\overrightarrow{\mathbf{e}}_{m}, \overrightarrow{\mathbf{h}}_{m}\right)$ of a noncanonical aperture can be easily and efficiently obtained using the well-known techniques, such as the boundary integral resonant mode expansion [14] or the finite-element method (FEM) [15]. Furthermore, the integrations in (6) and (14) can be computed by means of numerical quadrature without adding a significant computational burden since the function $\Phi_{m}$ is independent of frequency and thus has to be computed just once.

For illustrative purposes, two examples of noncanonical apertures (modeled through FEM) are presented next. The first one is a quad-ridge aperture [see Fig. 8 (a)]. The directivity is maximized at $f_{0}=12 \mathrm{GHz}$, the copolar component is defined along $\hat{\mathbf{y}}$, and the main lobe has been scanned to the $\left(\theta_{0}, \phi_{0}\right)=\left(20^{\circ}, 0^{\circ}\right)$ direction. All 33 propagating modes of this case are considered in the maximization process. The obtained radiation pattern is shown in Fig. 9.

The second example consists in the maximization of the directivity from a completely arbitrary aperture, as shown in Fig. 8(b). As it can be seen, the shape of the aperture is an irregular polygon without any symmetry axis. For this example, the copolar direction is also defined along $\hat{\mathbf{y}}$, and the main lobe has also been scanned to the $\left(\theta_{0}, \phi_{0}\right)=\left(20^{\circ}, 0^{\circ}\right)$ direction. All 52 propagating modes of this

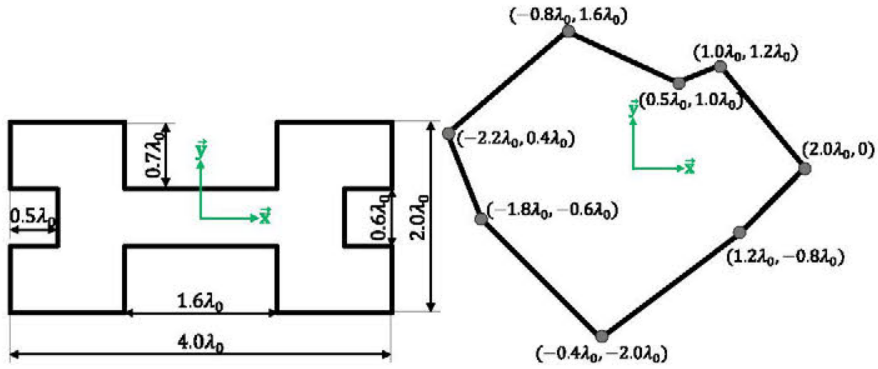

(a)

(b)

Fig. 8. Profile of the noncanonical apertures studied in the examples. (a) Quad-ridge. (b) Arbitrary.

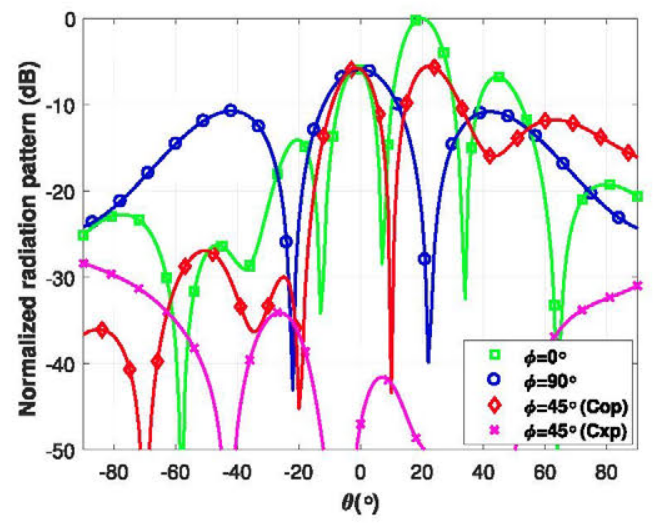

Fig. 9. Normalized radiation pattern of a linearly polarized quad-ridge radiating aperture. The main lobe has been scanned to $\left(\theta_{0}, \phi_{0}\right)=\left(20^{\circ}, 0^{\circ}\right)$. $D_{0}=23.54 \mathrm{dBi}$.

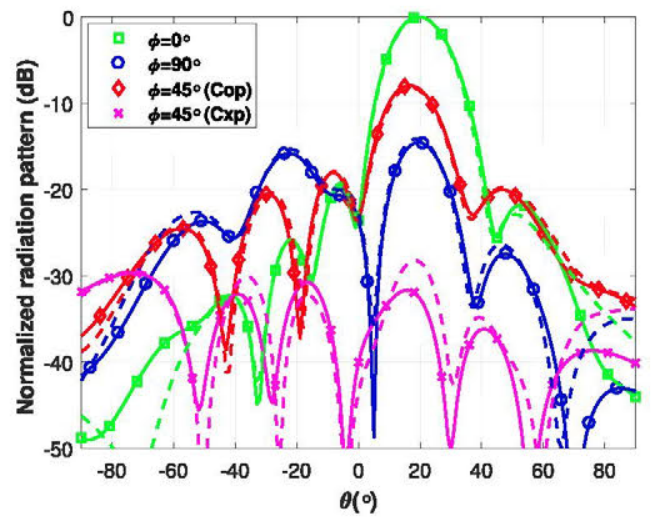

Fig. 10. Normalized radiation pattern of a linearly polarized completely arbitrary radiating aperture. The main lobe has been scanned to $\left(\theta_{0}, \phi_{0}\right)=$ $\left(20^{\circ}, 0^{\circ}\right)$. Solid line: this communication, with $D_{0}=19.81 \mathrm{dBi}$. Dashed: CST, with $D_{0}=20.07 \mathrm{dBi}$.

case are considered during the optimization process. The obtained radiation pattern can be found in Fig. 10. The radiation pattern has also been computed using CST Microwave Studio, and the results obtained by this tool have been superimposed over the ones from this communication. It can be seen how they are in good agreement. In case sidelobe and/or cross-polar radiation needs to be controlled, it is interesting to note that additional constraints regarding these levels can also be included in the formulation in future works to yield optimal patterns [8], [16], [17]. 


\section{CONCLUSION}

In this communication, a closed-form expression for the directivity maximization from radiating apertures with general shape has been derived. Given a specified aperture geometry and the number of modes at this aperture, the proposed technique computes the modal amplitudes that maximize the directivity in a desired direction at the specified operating frequency.

The analytical expression for directivity maximization has been compared, under the same assumptions, with those provided by the previous work, yielding the same analytical results for the considered cases, using a different derivation. Moreover, additional numerical examples considering different main lobe directions, aperture geometries, and polarizations have been presented in order to demonstrate the potential of the proposed technique formulation.

Finally, it should be highlighted that the presented formulation is formally guaranteed to achieve the global maximum directivity value because of its convex nature (ratio of two Hermitian forms). Therefore, this communication provides a way to compute the theoretical upper limit for this figure of merit in aperture antenna design.

\section{REFERENCES}

[1] C. Balanis, Antenna Theory and Design. Hoboken, NJ, USA: Wiley, 2015.

[2] L. Shafai, S. K. Sharma, and S. Rao, Handbook of Reflector Antennas and Feed Systems: Feed Systems (Artech House Antennas and Propagation Library), vol. 2. Norwood, MA, USA: Artech House, 2013.

[3] A. Bhattacharya and R. Vaughan, "Design of simple array antennas for high directivity," in Proc. IEEE Can. Conf. Elect. Comput. Eng. (CCECE), May 2016, pp. 1-4.

[4] J. Guo, S. Liao, Q. Xue, and S. Xiao, "Planar aperture antenna with high gain and high aperture efficiency for 60-Ghz applications," IEEE Trans. Antennas Propag., vol. 65, no. 12, pp. 6262-6273, Dec. 2017.
[5] A. K. Bhattacharyya and G. Goyette, "A novel horn radiator with high aperture efficiency and low cross-polarization and applications in arrays and multibeam reflector antennas," IEEE Trans. Antennas Propag., vol. 52, no. 11, pp. 2850-2859, Nov. 2004.

[6] F. Gantmacher, Theory Of Matrices, vol. 1. New York, NY, USA: Chelsea, 1959.

[7] D. K. Cheng, "Optimization techniques for antenna arrays," Proc. IEEE, vol. 59, no. 12, pp. 1664-1674, Dec. 1971.

[8] J. Corcoles, M. A. Gonzalez, and J. Rubio, "Multiobjective optimization of real and coupled antenna array excitations via primaldual, interior point filter method from spherical mode expansions," IEEE Trans. Antennas Propag., vol. 57, no. 1, pp. 110-121, Jan. 2009.

[9] R. E. Collin and IEEE Antennas and Propagation Society, Field Theory of Guided Waves (IEEE/OUP Series on Electromagnetic Wave Theory). Piscataway, NJ, USA: IEEE Press, 1991.

[10] W. L. Stutzman and G. A. Thiele, Antenna Theory and Design. Hoboken, NJ, USA: Wiley, 1998.

[11] A. Ludwig, "The definition of cross polarization," IEEE Trans. Antennas Propag., vol. 21, no. 1, pp. 116-119, Jan. 1973.

[12] IEEE Standard for Definitions of Terms for Antennas, IEEE Standard 145-2013 (Revision IEEE Std 145-1993), Mar. 2014, pp. 1-50.

[13] S. J. Orfanidis, An Introduction to Electromagnetic Wave Propagation \& Antennas, 2002. [Online]. Available: www.ece.rutgers.edu/ orfanidi/ewa

[14] G. Conciauro, R. Sorrentino, and M. Guglielmi, Advanced Modal Analysis: CAD Techniques for Waveguide Components and Filters. Hoboken, NJ, USA: Wiley, 2000.

[15] G. Pelosi, R. Coccioli, and S. Selleri, Quick Finite Elements for Electromagnetic Waves. Norwood, MA, USA: Artech House, 2009.

[16] T. Isernia and G. Panariello, "Optimal focusing of scalar fields subject to arbitrary upper bounds," Electron. Lett., vol. 34, no. 2, pp. 162-164, Jan. 1998.

[17] O. M. Bucci, T. Isernia, and A. F. Morabito, "Optimal synthesis of directivity constrained pencil beams by means of circularly symmetric aperture fields," IEEE Antennas Wireless Propag. Lett., vol. 8, pp. 1386-1389, 2009 